\section{Distributed temperature sensor system based on Raman scattering using correlation-codes}

M.A. Soto, P.K. Sahu, S. Faralli, G. Bolognini, F. Di Pasquale, B. Nebendahl and C. Rueck

A distributed sensor system employing spontaneous Raman scattering with use of correlation-coding techniques and a single-detector scheme is discussed and experimentally characterised. A sensing distance of up to $8 \mathrm{~km}$ is achieved with high spatial and temperature resolutions; use of correlation-coding significantly reduces measurement time and allows use of low-power laser sources.

Introduction: Fibre-based distributed temperature sensor (DTS) systems have been intensively studied for several years, because of the advantages they can provide compared to conventional sensors, and to optical fibre multiplexed sensor systems (such as grating-based sensors); most common applications include pipeline monitoring (for oil and gas transportation), oil well monitoring and power cables as well as fire detection systems [1].

Spontaneous Raman scattering effect is commonly exploited to implement DTS systems [2]. In Raman-based schemes, the ratio of Raman antiStokes (AS) line to Stokes line intensities is usually used for temperature monitoring, since it allows for measurements which are independent of major fibre loss effects and loss changes owing to fibre ageing and other effects. Temperature sensing along the fibre is then generally achieved through optical time domain reflectometry (OTDR), where light pulses are coupled into the fibre, and backscattered Stokes and AS light are detected. One drawback of such implementation schemes is that, owing to low Raman backscattering power, high peak power and long measurement time must be used to ensure good spatial and temperature resolution. Coding techniques can be used in OTDR to overcome the resolutionrange trade-off and improve signal-to-noise ratio (SNR) [3].

In this Letter we deal with the implementation and characterisation of a novel distributed Raman-based DTS (Agilent DTS N4385A/ N4386A proprietary technology) using for first time complementary-correlation (CC) coded OTDR techniques with a single photodiode receiver scheme. Use of correlation coding allowed overcoming of the resolution-range trade-off by enhancing SNR, resulting in a high-performance DTS over $8 \mathrm{~km}$ multimode (MM) sensing fibre. The newly proposed single photodetector receiver scheme allows for high measurement repeatability over a wide temperature range. Experimental characterisation has shown temperature sensing over $4 \mathrm{~km}(8 \mathrm{~km})$ of MM fibres with $1 \mathrm{~m}(3 \mathrm{~m})$ spatial resolution and $0.3 \mathrm{~K}$ temperature resolution.

Theory: Raman DTSs are usually implemented through OTDR technique by measuring the intensity of backward-propagating radiation over fibre length. In particular, the intensity ratio between temperature-dependent Stokes and AS is used to obtain reliable temperature estimations, reducing the impact of fibre loss; the temperature dependence of the ratio can be expressed as:

$$
\frac{I_{A S}}{I_{S}} \propto \exp \left(-\frac{h \Delta v_{R}}{k T}\right)
$$

where $k$ is the Boltzmann constant, $T$ the absolute temperature, $h$ the Planck constant, and $\Delta v_{R}$ the frequency separation between Raman AS/Stokes and Rayleigh scattering light. Proper correction for $\lambda$-dependent fibre- and receiver-parameters is also required for absolute $T$ calculation; this can be done by performing suitable real-time fibre calibration, allowing for reliable and accurate temperature evaluation.

Among pulse coding techniques, correlation-based coding can be effectively used to overcome the resolution-range trade-off and to increase SNR, similar to the OTDR case [4], thus enhancing DTS performance. The basic idea behind correlation techniques is to spread the signal in the time domain, and to reconstruct the fibre impulse response by correlating the detected signal with a probe; it is then possible to avoid the onset of nonlinear effects and to improve attained SNR. Among different coding schemes, CC-Golay codes [4] have all required features to be used in direct-detection DTS schemes.

The proposed Raman DTS system, exploiting for first time correlation coding, and specifically CC-Golay codes, provides strongly enhanced performance. The coding gain $\left(G_{\text {cod }}\right)$ parameter quantifies the SNR enhancement provided by coding with respect to the single pulse case (for the same number of acquired traces). For Golay codes of codeword length $L, G_{\text {cod }}$ is given by $\sqrt{L / 2}$. Golay codes have the significant advantage that they allow SNR improvement in a DTS with use of only two CC-codeword pairs (independently of code length), consisting of a significant reduction of measurement time with respect to previously reported codes (e.g. simplex coding in [3]). The provided SNR improvement can be used for enhancing the sensing range, the spatial and temperature resolution, and for reducing measurement time. Finally, use of coding in our work has permitted employing low-power semiconductor lasers with long operating life, low energy consumption and reduced user hazards when compared, e.g., to standard high-power pulsed lasers used in common OTDR-based DTS (e.g. YAG laser).

Setup: Fig. 1a shows the block diagram of the experimental setup used to implement the Raman-based DTS. The OTDR source is provided by an extremely low average output power external cavity laser $\left(P_{A V}=17 \mathrm{~mW}\right.$, class $1 \mathrm{M}, 1064 \mathrm{~nm}$, pulse duration proportional to spatial resolution setting); Raman-backscattered light from the sensing fibre is directed into an optical receiver block as shown in Fig. $1 b$. Note that the low power level of the laser source permits avoiding nonlinear optical effects, and, moreover, laser power stability permits avoiding spurious effects in temperature traces owing to the decoding process. This unit is composed of $\lambda$-selective filters (WFs), separating the Stokes and AS components, mirrors (M) and an optical shutter allowing for desired $\lambda$ selection, combined with a single photodiode (PD) receiver. A single receiver scheme can be used for both AS and Stokes measurement in a configuration similar to timedomain multiplexing detection. Use of a single receiver with respect to the well-known double receiver design allows for high stability and repeatability, since receiver offset, gain and bandwidth characteristics are the same during acquisition of both traces. For measurement time optimisation two separate processors are used for temperature calculation and data processing (e.g. correlation/decorrelation). The sensing fibre $(4430 \mathrm{~m})$ used in scheme A (Fig. 1c) consists of five fibre spools of graded-index MMF $(50 / 125 \mu \mathrm{m})$ (a different temperature can be set for each spool). In Fig. $1 c$ scheme B ( $8500 \mathrm{~m}$ of MMF) is used to obtain data for temperature resolution over longer distances.

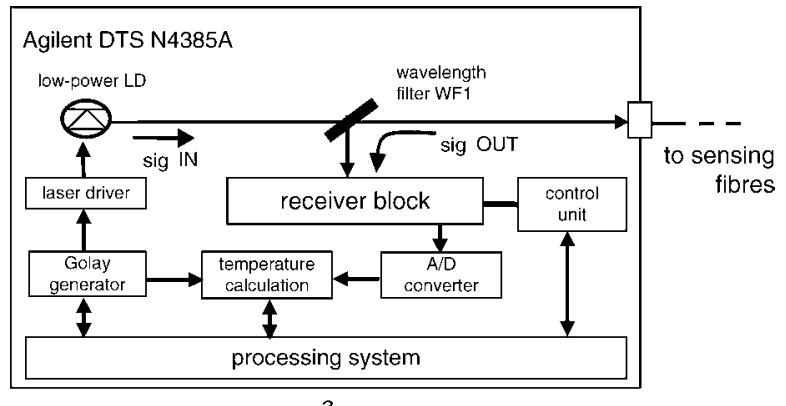

a

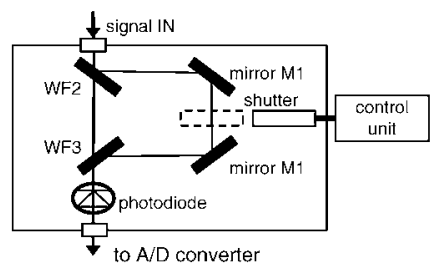

$b$

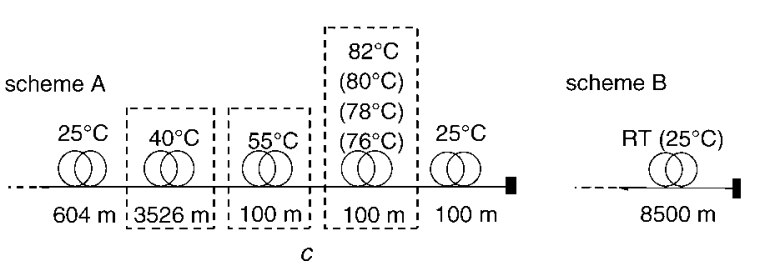

Fig. 1 Schematic of DTS, transmitter and single-photodiode receiver (Fig. 1a); details of single-photodiode receiver block (Fig. 1b); setup of different sensing fibre schemes used in experiments (Fig. 1c)

Results: Temperature measurements for the new correlation-based DTS system have been carried out for a set of different conditions. To 
characterise DTS performance at medium sensing range $(\sim 4 \mathrm{~km})$ we changed the temperature of $100 \mathrm{~m}$ of fibre in scheme A (coil 4 in Fig. 1 c) in $2 \mathrm{~K}$ steps, in the range $76-82^{\circ} \mathrm{C}$. The results obtained after $10 \mathrm{~min}$ of measurement time are shown in Fig. 2. The difference between the set and the measured temperature step was less than $0.3 \mathrm{~K}$. The temperature resolution $\left(\Delta T_{R E S}\right)$ of the DTS was calculated as an exponential fit of the standard deviation (STD) from measured temperature distribution. The STD and $\Delta T_{R E S}$ with a spatial resolution of $1 \mathrm{~m}$ (for $1 \mathrm{~min}$ and $10 \mathrm{~min}$ measurement time, respectively) are shown in Fig. 3 (6.25 ns single pulse duration allowing for $1 \mathrm{~m}$ resolution up to the fibre end). Fig. $3 a$ shows $\Delta T_{R E S}$ for scheme A; at around $4 \mathrm{~km}$ sensing length they are about 0.86 and $0.30 \mathrm{~K}$, respectively. Performance of the DTS up to $8 \mathrm{~km}$ length is shown in Fig. $3 b$ based on scheme B (7.45 and $2.62 \mathrm{~K}) ; \Delta T_{R E S}$ values shown in Fig. $3 b$ are worse than in Fig. $3 a$ at the same fibre position owing to lower repetition rate of the Golay sequences in longer fibres for the same total acquisition time. By changing the spatial resolution settings to 1.5 and $3 \mathrm{~m}$, achieved $\Delta T_{R E S}$ values at $8 \mathrm{~km}$ after $10 \mathrm{~min}$ of averaging time are found to be 0.65 and $0.27 \mathrm{~K}$, respectively, over a wide fibre temperature range $\left(-20-60^{\circ} \mathrm{C}\right)$.

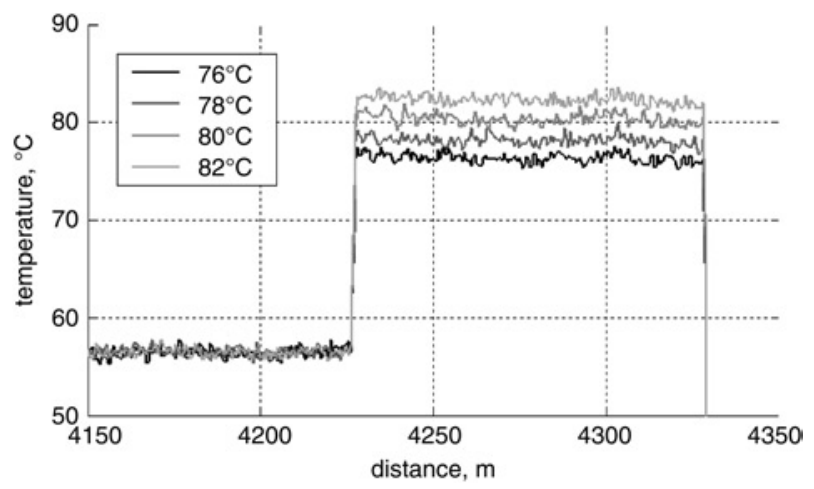

Fig. 2 Temperature distribution with $100 \mathrm{~m} \mathrm{MMF}$ placed at different temperatures
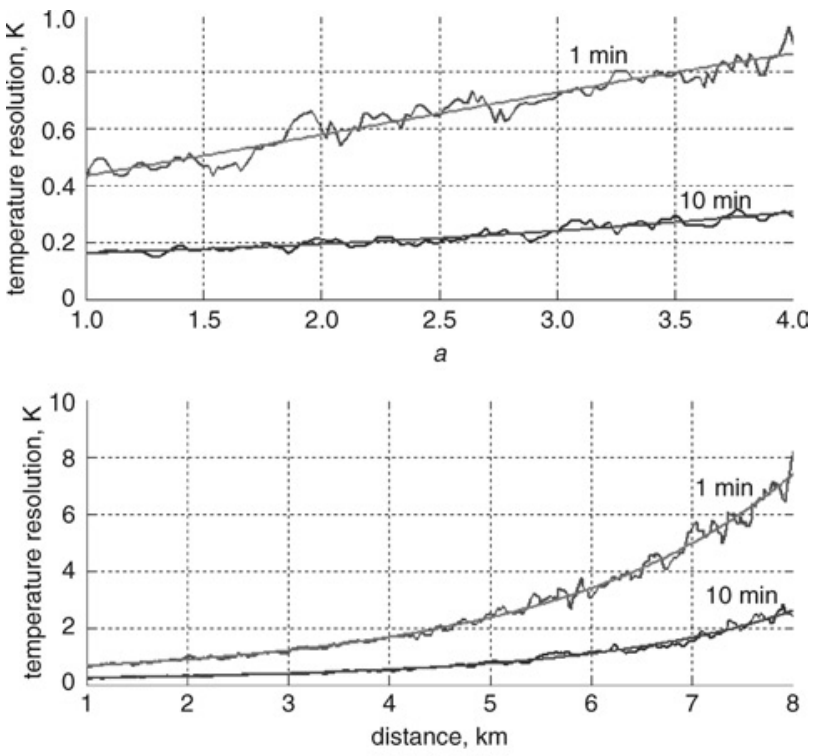

b

Fig. 3 Root mean square temperature resolution (and exponential fit) against fibre length for 4 and $8 \mathrm{~km}$ sensing distance (measurement time of 1 and $10 \mathrm{~min}$ )

a $4 \mathrm{~km}$ sensing distance

$b 8 \mathrm{~km}$ sensing distance
The repeatability and stability of the above DTS system, which are important owing to changing DTS conditions in real applications, have been investigated over a broad operating temperature range of DTS itself $\left(-10-60^{\circ} \mathrm{C}\right)$; the measured repeatability at about $4 \mathrm{~km}$ was smaller than $1 \mathrm{~K}$, for constant measurement conditions $(0.5 \mathrm{~m}$ sampling interval, $1 \mathrm{~m}$ spatial resolution setting, $10 \mathrm{~min}$ measurement time). By changing spatial resolution settings to $3 \mathrm{~m}$, repeatability was smaller than $0.2 \mathrm{~K}$. Note that this result is mainly attained thanks to the single receiver scheme, avoiding different behaviour of each receiver in a double receiver scheme.

Conclusion: A novel distributed sensor system based on spontaneous Raman scattering with Golay coding has been discussed and characterised, for applications up to $8 \mathrm{~km}$ sensing distance. CC-coding techniques and use of multimode fibres in a single receiver scheme allowed for high spatial and temperature resolution sensing with high repeatable measurement using low power semiconductor lasers.

(C) The Institution of Engineering and Technology 2007

6 April 2007

Electronics Letters online no: 20070990

doi: 10.1049/el:20070990

M.A. Soto, P.K. Sahu, S. Faralli, G. Bolognini and F. Di Pasquale (Scuola Superiore Sant'Anna, via G. Moruzzi 1, Pisa 56124, Italy)

E-mail: gabriele.bolognini@cnit.it

B. Nebendahl and C. Rueck (Agilent Technologies R\&D and Marketing GmbH \& Co. KG, Germany)

\section{References}

1 Grattan, L.S., and Meggitt, B.T. (eds), 'Optical fiber sensor technology: advanced applications Bragg gratings and distributed sensors' (Springer, London, 2000)

2 Ahangrani, J M., et al.: 'Spontaneous Raman scattering in optical fibers with modulated probe light for distributed temperature Raman remote sensing', J. Lightwave Technol., 1999, 17, pp. 1379-1391

3 Bolognini, G., et al.: 'Improved performance in Raman-based distributed temperature sensing with coded OTDR and discrete Raman amplification'. OFS 2006 Conf. Tech. Dig., Cancun, Mexico, p. ThE45

4 Nazarathy, M., et al.: 'Complementary correlation OTDR with three codewords', Electron. Lett., 1990, 26, (1), pp. 70-71 